\title{
Heat of Mixing of Polybutadiene and Benzene
}

\section{Ralph S. Jessup}

\begin{abstract}
Measurements have been made of the heat of mixing of polybutadiene and benzene at $26.9^{\circ} \mathrm{C}$ by means of a Bunsen-type calorimeter, in which diphenyl ether is the calorimetric medium. The results of these measurements can be represented by the empirical equation

$$
\Delta H^{M}=12.0 v_{1} v_{2},
$$

where $\Delta H^{\mathrm{M}}$ is heat of mixing in joules per $\mathrm{cm}^{3}$, and $v_{1}$ and $v_{2}$ are volume fractions of solvent and polymer, respectively. The data have been combined with previously reported data on the activity of the solvent in this system to obtain values of $\Delta \bar{S}_{1}$, the partial molal entropy of dilution. The curve of $\Delta \bar{S}_{1} / R v_{2}^{2}$ versus $v_{2}$ for this system lies above the calculated curve of $\Delta \bar{S}_{1}^{*} / R v_{2}^{2}$, where $\Delta \bar{S}_{1}^{*}$ is the "configurational entropy" of dilution, and also lies above the corresponding experimental curves for several other polymer-solvent systems.
\end{abstract}

\section{Introduction}

This paper presents results of measurements of heat of mixing of polybutadiene with benzene. These results supplement data previously reported $[1]^{1}$ on the activity of the solvent in this system. The two sets of data have been combined to yield values of partial molal entropies of dilution.

\section{Materials}

The sample of polybutadiene used, and its purification, including removal of solvent and permanent gases, were described in reference [1]. Reagent grade benzene was purified by fractional freezing as described previously $[1,4]$, and was further purified by removal of dissolved permanent gas as described in section 3.2 of this paper. The density at $25^{\circ} \mathrm{C}$ of the purified benzene before removal of dissolved gas was $0.87334 \mathrm{~g} / \mathrm{cm}^{3}$, as compared with the value $0.87368 \mathrm{~g} / \mathrm{cm}^{3}$ reported in the literature [3].

\section{Apparatus and Method}

\subsection{Apparatus}

The measurements of heat of mixing were made at $26.9^{\circ} \mathrm{C}$ by means of a Bunsen-type calorimeter in which diphenyl ether is the calorimetric medium. Heat absorbed in the mixing process causes partial freezing of the diphenyl ether, with a consequent change in volume. This change in volume is measured by the displacement of a mercury meniscus in a graduated capillary connected to a pool of mercury in the bottom of the calorimeter. The calorimeter has been described previously [2] and only a brief description will be given here.

The calorimeter, as used in measurements of heats of mixing of two liquids, and a buret for introducing measured quantities of one component of the mixture are shown schematically in figure 1. The mixing chamber $\mathrm{F}$, the cylindrical copper pieces $\mathrm{V}$, the conical copper piece $K$, and the connecting tubing up to a union (not shown in fig. 1 ) in the tube $J$ just above $\mathrm{K}$ form a unit that can be lifted out of the central

\footnotetext{
1 Figures in brackets indicate the literature references at the end of this paper.
}

well of the calorimeter. This unit can be replaced by a similar unit with an electric heater for calibration purposes in place of the mixing vessel [2]. The conical copper piece $\mathrm{K}$ fits into a conical hole in the copper jacket cover and forms a part of this cover.

For the present measurements, the calorimeter was modified by (1) substituting a smaller buret and a smaller mixing chamber for those shown in figure 1 , (2) eliminating the stirrer ( $\mathrm{Z}$, fig. 1), and (3) sealing off the mixing chamber at the point $Y$ from the tube through which the stirrer shaft passes.

The buret used had a capacity of a little over $1 \mathrm{~cm}^{3}$, and could be read directly to $0.01 \mathrm{~cm}^{3}$ and by interpolation to $0.001 \mathrm{~cm}^{3}$. The mixing chamber had a capacity of $6.3 \mathrm{~cm}^{3}$. The mixing chamber also contained a shallow cup (not shown in fig. 1) into which liquid solvent admitted to the mixing chamber dropped, so that solvent came in contact with the polymer only in the gas phase.

In a measurement of heat of mixing, the solvent flows from the buret, where the volume of the liquid is measured, to the mixing chamber through the copper tube $J$. A part of this tube (not shown in fig. 1) is wound into a spiral and soldered to the jacket cover to insure that the liquid is at the jacket temperature before it enters the calorimeter. After leaving the spiral the liquid continues through the tubes $J$ and $L$ to the valve $A$.

The valve A (fig. 1) was originally a small needle valve, which was found to be unsuitable because appreciable and irregular quantities of heat were generated in opening and closing it. It was therefore replaced by a different type of valve illustrated in figure 2. The point of the valve needle $\mathrm{C}$ is enclosed in a Teflon sheath $\mathrm{S}$ as shown, and the pointed end of this sheath is pressed against the valve seat by a helical spring $W$. This spring is located in a monel metal tube $\mathrm{M}$ which projects above the conical copper piece $\mathrm{K}$.

The upper end of the tube $\mathrm{M}$ is soldered to the stuffing box B (fig. 2) and its lower end is soldered to the conical copper piece K. Also soldered to the copper piece $\mathrm{K}$ is the monel tube $\mathrm{L}$ which leads to the valve $A$. The upper end of the spring $W$ presses against the nut $\mathrm{N}$ at the bottom of the stuffing box, the nut being held in place by the force exerted by the nut $\mathrm{H}$ and transmitted through the packing 

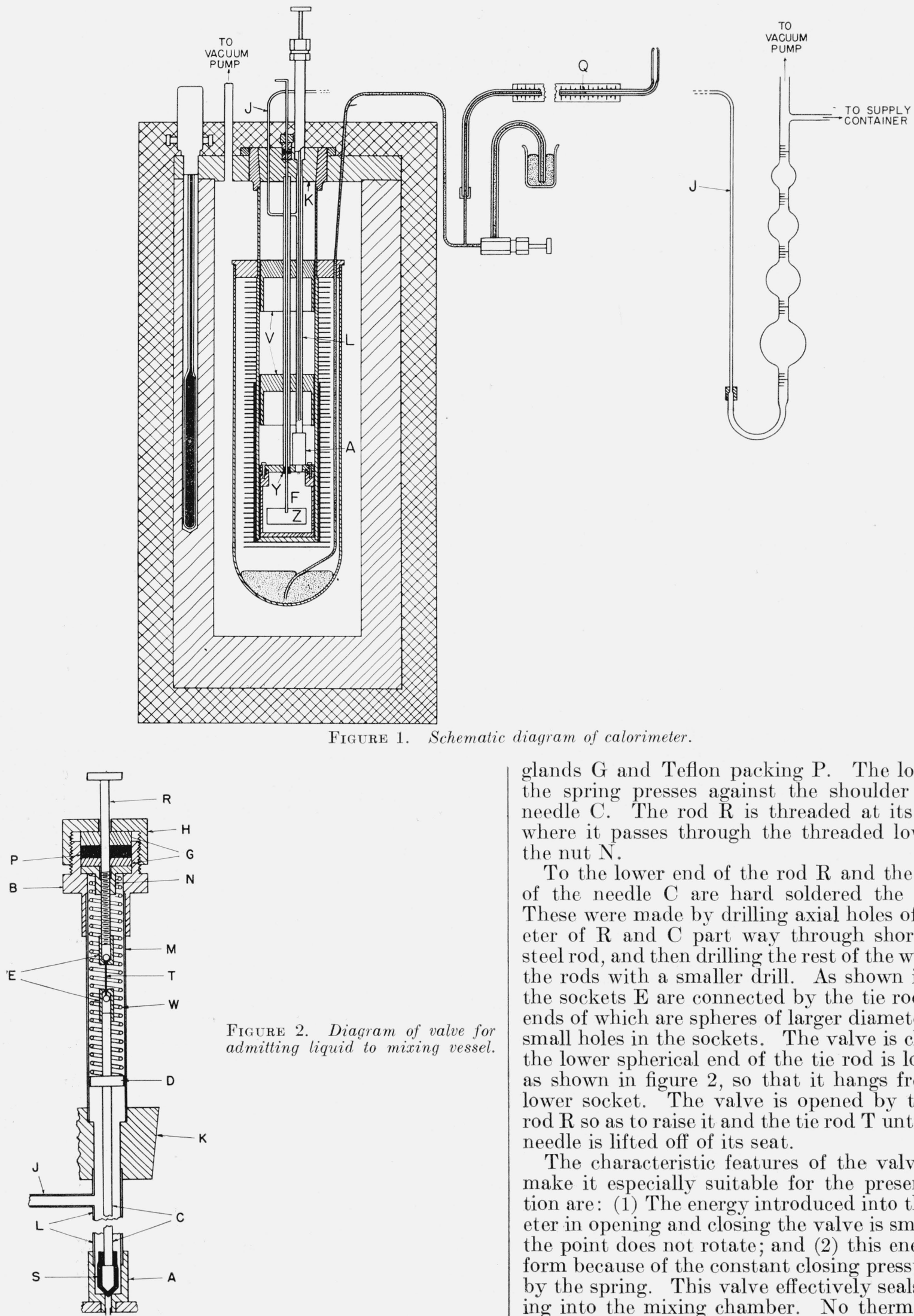

Figure 2. Diagram of valve for admitting liquid to mixing vessel.

glands $\mathrm{G}$ and Teflon packing $\mathrm{P}$. The lower end of the spring presses against the shoulder $\mathrm{D}$ on the needle $\mathrm{C}$. The rod $\mathrm{R}$ is threaded at its lower end where it passes through the threaded lower end of the nut $\mathrm{N}$.

To the lower end of the rod $\mathrm{R}$ and the upper end of the needle $\mathrm{C}$ are hard soldered the sockets $\mathrm{E}$. These were made by drilling axial holes of the diameter of $\mathrm{R}$ and $\mathrm{C}$ part way through short pieces of steel rod, and then drilling the rest of the way through the rods with a smaller drill. As shown in figure 2, the sockets $\mathrm{E}$ are connected by the tie rod T, at the ends of which are spheres of larger diameter than the small holes in the sockets. The valve is closed when the lower spherical end of the tie rod is low enough, as shown in figure 2, so that it hangs freely in the lower socket. The valve is opened by turning the $\operatorname{rod} \mathrm{R}$ so as to raise it and the tie rod $\mathrm{T}$ until the valve needle is lifted off of its seat.

The characteristic features of the valve A which make it especially suitable for the present application are: (1) The energy introduced into the calorimeter in opening and closing the valve is small because the point does not rotate; and (2) this energy is uniform because of the constant closing pressure exerted by the spring. This valve effectively seals the opening into the mixing chamber. No thermal effect of 
repeatedly opening and closing the valve was detected in blank experiments.

In measurements of heat of mixing the tubes $\mathrm{J}$, L, and M (figs. 1 and 2) are completely filled with liquid, and each time the valve $A$ is closed the rod $R$ (fig. 2) is brought to the same position so that the volume of liquid in these tubes is always the same when the buret is read. There was no appreciable change in temperature of this liquid between the time of reading the buret before and after introducing liquid into the mixing chamber.

\subsection{Experimental Method}

The method of measurement consists essentially in adding a measured volume of solvent to a known mass of polymer (or polymer-solvent mixture) in the mixing chamber in the calorimeter, and observing the resulting thermal effect as indicated by the change in position of the mercury meniscus in the graduated capillary Q (fig. 1). Because it was not feasible to stir the mixture, which was very viscous in the composition range covered, the polymer sample was placed in the mixing chamber in such a manner as to promote rapid attainment of equilibrium between polymer and solvent. The sponge-like polymer was cut into fairly thin sheets which were separated in the mixing chamber by metal gauze, so that solvent vapor could circulate freely between the polymer sheets. As indicated in section 3.1, solvent came in contact with the polymer (or mixture) only as vapor. Experience in measurements of vapor pressure in this system [1] indicated that the approach to equilibrium under the conditions existing in the mixing chamber should be sufficiently rapid to permit reasonably accurate calorimetric measurements of heat of mixing. This is confirmed by the actual results of the calorimetric measurements, which indicated that equilibrium was attained in time intervals ranging from $30 \mathrm{~min}$ in mixtures of low benzene content to $90 \mathrm{~min}$ for mixtures containing approximately 50 percent benzene.

The procedure for making measurements of heat of mixing is as follows: The mixing chamber unit is removed from the calorimeter and a mantle of solid diphenyl ether is frozen around the outside of the central well as described previously [2].

The mixing chamber, detached from the remainder of the unit, is weighed first with only the metal gauze in it, and again after introducing about $1 \mathrm{~g}$ of polybutadiene. The mixing chamber is then attached to the remainder of the unit and connected through the tubes $\mathrm{L}$ and $\mathrm{J}$, by means of the union in $J$, to a vacuum system. Solvent and permanent gases are removed from the polymer by prolonged evacuation to a pressure of less than $10^{-4} \mathrm{~mm}$ while heating to temperatures increasing gradually to a maximum of about $95^{\circ} \mathrm{C}$. During this process most of the phenyl-beta-napthylamine used as an antioxidant sublimes from the sample. The valve $\mathrm{A}$ is then closed, and the mixing-chamber unit is replaced in the calorimeter and connected through the system of tubing described in section 3.1 to the buret (fig. 1 ). The observed mass of the sample was corrected for loss of phenyl-beta-naphthylamine and dissolved gases.

A supply of the solvent is placed in a container consisting of a glass bulb to which a brass valve is attached, and the container is connected through the valve to a vacuum pump. Air is removed from the liquid by freezing it in liquid air and evacuating the container, and by removal of part of the liquid by evacuation at room temperature. This procedure is repeated a number of times until, with the vacuum pump shut off, the pressure in the system after freezing the liquid is of the order of $10^{-5} \mathrm{~mm}$ of mercury. The valve on the container is then closed, the container disconnected from the vacuum system, inverted, and attached to the buret (fig. 1).

The buret and connecting tubing up to valve $\mathrm{A}$ (figs. 1 and 2), are evacuated until the pressure is reduced to less than $10^{-5} \mathrm{~mm}$ of mercury. The vacuum pump is then shut off and the valve on the supply container is opened, allowing the liquid to flow into the buret. The container is then disconnected from the buret, permitting air to flow into the buret above the liquid surface, the line to the vacuum pump is broken, and additional liquid introduced into the buret to bring the meniscus up to the zero graduation mark. It is believed that this procedure effectively eliminates the possibility of gas bubbles in the line between the buret and the mixing chamber, or in the tube M (fig. 2).

Before making a calorimetric measurement the jacket thermostat is adjusted so as to bring the temperature of the jacket within $0.001 \mathrm{deg} C$ of that of the calorimeter. After allowing a reasonable time for the system to come to a steady state, readings are made at 10 -min intervals of (1) the difference in temperature between calorimeter and jacket to about $0.0001 \mathrm{deg}$, (2) room temperature to the nearest $0.1 \mathrm{deg}$, and (3) the position of the mercury meniscus in the graduated capillary Q (fig. 1) to the nearest $0.1 \mathrm{~mm}(0.04 \mathrm{j}$ or $0.01 \mathrm{cal})$. These measurements are continued for a sufficient length of time (30 to $60 \mathrm{~min}$ ) to insure that the capillary reading is constant or changing at a uniform rate. The valve A (figs. 1 and 2) is then opened and liquid permitted to flow from the buret into the mixing vessel. The valve is closed again when the desired amount of liquid has been introduced into the mixing chamber, as indicated by the change in reading of the buret. The readings (1), (2), and (3) above are continued at 10 -min intervals until the capillary reading has been constant or changing at a uniform rate for 30 to $50 \mathrm{~min}$. The same procedure is followed in subsequent experiments, the final "rating period" of each experiment being taken as the initial rating period of the next. The volumes of liquid added in successive experiments were approximately $0.1,0.1,0.2,0.4$, and $0.3 \mathrm{~cm}^{3}$. These volumes were measured at approximately $25.5^{\circ}, \mathrm{C}$. and were reduced to the corresponding volumes at ${ }^{\circ} 26.9 \mathrm{C}$. 


\subsection{Calculation of Results}

The observed change in reading of the capillary in each experiment is multiplied by the calibration factor, $4.145 \mathrm{j} / \mathrm{cm}$ [2], to give the observed thermal effect. This is corrected for: (1) Thermal leakage determined from the rate of change of capillary reading in the rating periods preceding and following the "mixing period" in each experiment, (2) the work done by the atmosphere in forcing the liquid through the valve A into the evacuated mixing chamber, (3) the change in internal energy of the liquid introduced in each experiment in going from the buret at atmospheric pressure to nearly zero pressure in the mixing vessel, and (4) the change in energy of the vapor in the mixing vessel due to the difference between the vapor volumes and vapor densities at the beginning and end of the experiment. The correction (1) for thermal leakage ranged from zero to about 10 percent of the heat measured in a given experiment. The corrections (2) and (3) amount, respectively, to approximately -0.10 and $+0.04 \mathrm{j} / \mathrm{cm}^{3}$ of liquid added. Correction (4) was calculated from the known vapor volumes at the beginning and end of the experiment, the vapor densities as determined from the activity-composition relation for this system [1], and the energy of vaporization of benzene. The magnitude of this correction ranged from 8 to 20 percent of the heat measured.

The result of an experiment calculated as described above is, strictly speaking, the intrinsic energy of mixing at nearly constant pressure. This is practically equal to the heat (or enthalpy) of mixing at constant pressure, and is so designated in this paper.

\section{Results}

The observed values of heat of mixing are compared in table 1 with values calculated from the equation ${ }^{2}$

$$
\Delta H^{M}=12.00 v_{1} v_{2},
$$

where $\Delta H^{M}$ is heat of mixing in joules per $\mathrm{cm}^{3}$ of mixture, and $v_{1}$ and $v_{2}$ are volume fractions of solvent and polymer, respectively. The maximum difference between observed and calculated values corresponds to about $0.23 \mathrm{j}(0.06 \mathrm{cal})$ in a calorimetric measurement. This is somewhat greater than the maximum deviation from the mean $(0.15 \mathrm{j}$ or $0.04 \mathrm{cal})$ in the calibration experiments [2], but larger calorimetric errors are to be expected in the present measurements because of the longer time required to reach equilibrium. ${ }^{3}$

\footnotetext{
${ }^{2}$ Equation (1) is of the same form as the well-known van Laar equation, but the constant factor 12.0 was determined empirically from the data. The solubility parameters [9] for benzene and polybutadiene yield the value 2.0 for this constant factor.

3 Better agreement between observed and calculated values could be obtained by using an equation of the form $\Delta H^{M}=A v_{1} v_{2}\left(1+B v_{1}\right)$, but it is not believed that the data are sufficiently accurate to justify this refinement.
}

In table 2 are given values of partial molal heat of dilution $\left(\Delta \bar{H}_{1}\right)$ derived from eq $(1)$; partial molal Gibbs free of energy of dilution $\left(\Delta \bar{G}_{1}\right)$, derived from the relation $\Delta \bar{G}_{1}=R T \ln a_{1}$, with

$$
\ln a_{1}=\ln v_{1}+v_{2}+0.27 v_{2}^{2}+0.06 v_{2}^{3} ;
$$

and partial molal entropy of dilution $\left(\Delta \overline{S_{1}}\right)$ calculated from the relation

$$
\Delta \bar{S}_{1}=\left(\Delta \bar{H}_{1}-\Delta \bar{G}_{1}\right) / T .
$$

Equation (2) above is eq (2) for the activity of the solvent in reference [1].

TABLE 1. Observed values of heat of mixing at $26.9^{\circ} \mathrm{C} \mathrm{com-}$ pared with values calculated from eq (1)

\begin{tabular}{|c|c|c|c|}
\hline & \multicolumn{2}{|c|}{ Heat of mixing, $\mathrm{j} / \mathrm{ml}$ of mixture } \\
$\begin{array}{c}\text { Volume frac- } \\
\text { tion benzene, } \\
v_{1}\end{array}$ & Observed & Calculated & $\mathrm{O}-\mathrm{C}$ \\
\cline { 1 - 1 } & & & \\
\cline { 1 - 2 } 0.1088 & 0.986 & 1.164 & -0.178 \\
.1668 & 1.633 & 1.668 & -.035 \\
.2756 & 2.407 & 2.397 & +.010 \\
.4146 & 2.890 & 2.913 & -.023 \\
.4904 & 3.096 & 3.000 & +.096 \\
\hline
\end{tabular}

\begin{tabular}{|c|c|c|c|c|}
\hline $\begin{array}{l}\text { Volume } \\
\text { fraction } \\
\text { polymer } v_{2}\end{array}$ & $\Delta \bar{H}_{1}$ & $\Delta \bar{G}_{1}$ & $T \Delta \overline{S_{1}}$ & $\Delta \bar{S}_{1}$ \\
\hline $\begin{array}{r}0.5 \\
.6 \\
.7 \\
.8 \\
.9 \\
1.0\end{array}$ & $\begin{array}{r}j / \text { mole } \\
269 \\
387 \\
527 \\
689 \\
871 \\
1,076\end{array}$ & $\begin{array}{r}j / \text { mole } \\
-295 \\
-514 \\
-876 \\
-1,512 \\
-2,844 \\
-\infty\end{array}$ & $\begin{array}{r}j / \text { mole } \\
564 \\
901 \\
1,403 \\
2,201 \\
3,715 \\
\infty\end{array}$ & $\begin{array}{c}j / \text { mole deg } \\
1.88 \\
3.00 \\
4.68 \\
7.34 \\
12.38 \\
\infty\end{array}$ \\
\hline
\end{tabular}

TABLE 2. Values of partial molal enthalpy, Gibbs free energy and entropy of dilution at $26.9^{\circ} \mathrm{C}$

Values of $\Delta \overline{S_{1}} / R v_{2}^{2}$ are plotted versus $v_{2}$ in figure 3 , where curve 1 represents the data of table 2 , curve 2 represents similar results for the system natural rubber-benzene [5], and curve 3 represents values of $\Delta \bar{S}_{1}^{*} / R v_{2}^{2}$, where $\Delta \bar{S}_{1}^{*}$ is the "configurational entropy" of dilution [6] calculated from the equation

$$
\Delta \bar{S}_{1}^{*}=-R\left[\ln v_{1}+v_{2}\right] .
$$

The curve 1 in figure 3 for the system polybutadiene-benzene is of the same general form as curve 3 , but lies considerably above it. It is also higher than curve 2 for the rubber-benzene system [5], and than curves [6, p. 518] for the polymersolvent systems polydimethylsiloxane $(\mathrm{M}=2850)$ benzene [7], polystyrene-toluene, and polystyrenebutanone [8], all three of which lie below curve 3 (fig. 3) by varying amounts. 


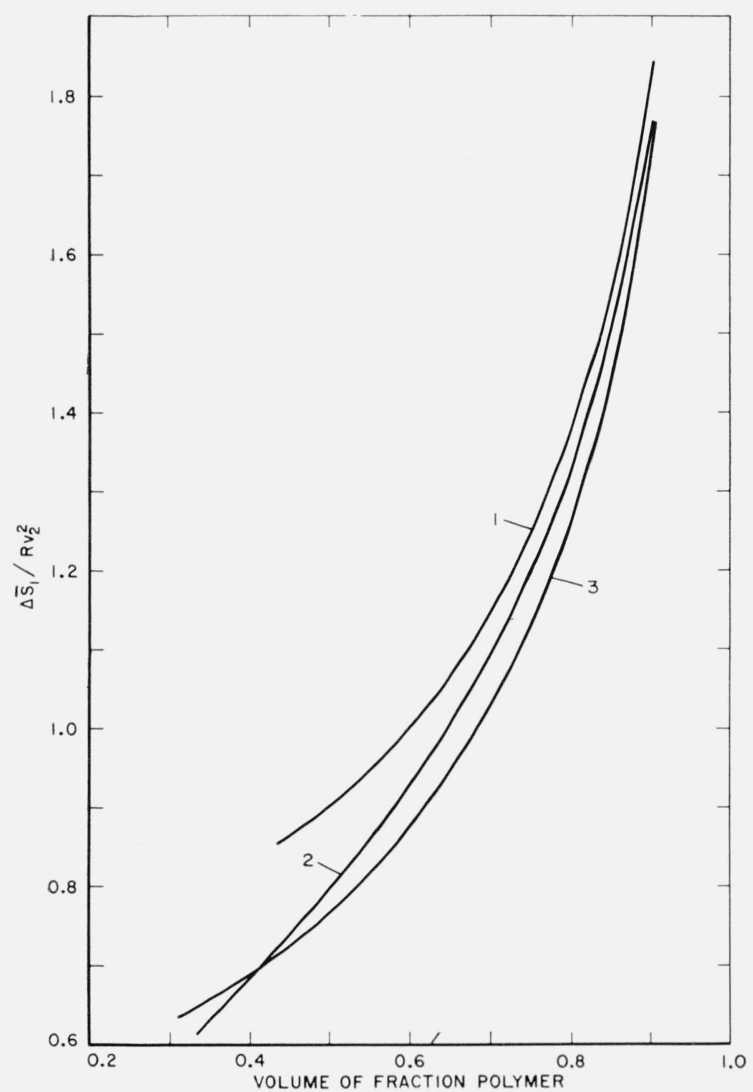

Figure 3. Comparison of observed entropy of dilution $\Delta \bar{S}_{1}$ in the system polybutadiene-benzene (curve 1) with that for natural rubber-benzene (curve 2) and with $\Delta \bar{S}_{1}^{*}$ calculated from eq (3).

\section{References}

[1] R. S. Jessup, J. Research NBS 60, 47 (1958) RP2820.

[2] R. S. Jessup, J. Research NBS 55, 317 (1955) RP2636.

[3] Selected values of properties of hydrocarbons, Table $5 \mathrm{a}$ Am. Petrol. Inst. Research Project 44, Carnegie Inst. of Technol. (1956).

[4] F. W. Schwab and E. Wichers, J. Research NBS 25, 747 (1940) RP1357.

[5] G. Gee and W. J. C. Orr, Trans. Faraday Soc. 42, 507 (1946).

[6] P. J. Flory, Principles of polymer chemistry, p. 512 (Cornell University Press, Ithaca, N. Y., 1953).

[7] M. J. Newing, Trans. Faraday Soc. 46, 613 (1950).

[8] C. E. H. Bawn, R. F. J. Freeman, and A. R. Kamalidden, Trans. Faraday Soc. 46, 677 (1950).

[9] J. H. Hildebrand and R. L. Scott, The solubility of nonelectrolytes, p. 387 (Reinhold Publishing Corporation, New York, N. Y., 1950).

Washington, July 23, 1958. 